\title{
COPD-Screening in der hausärztlichen Praxis mit einem Lungenfunktions-Schnellmessgerät
}

\author{
Use of a Lung Function Screening Device for Identifying Patients \\ at Risk for COPD in General Practice
}

Autoren

Institute
M. Müller ${ }^{1}$, H. Kögler², T. Glaab² ${ }^{2}$ T. Welte ${ }^{3}$

Pfizer, Med. Wissenschaft, Berlin

Boehringer Ingelheim, Med. Wissenschaft, Ingelheim

3 Medizinische Hochschule Hannover, Abt. Pneumologie, Hannover eingangen 14.9.2012 akzeptiert nach Revision 24. 9. 2012

\section{Bibliografie}

Dol http://dx.doi.org/ 10.1055/s-0032-1325791 Pneumologie 2012; 66: 645-649 (c) Georg Thieme Verlag KG Stuttgart · New York ISSN 0934-8387

\section{Korrespondenzadresse} Prof. Dr. Tobias Welte

Abteilung Pneumologie Medizinische Hochschule Hannover

Carl-Neuberg-Str.1 30625 Hannover Welte.Tobias@mh-hannover.de

\section{Zusammenfassung \\ $\nabla$}

Hintergrund: Screeningmaßnahmen können die Diagnosestellung einer chronisch obstruktiven Lungenerkrankung (COPD) erleichtern und helfen, Kosten und Zeit zu sparen. Wir untersuchten, ob die Messung mit einem LungenfunktionsSchnellmessgerät (Vitalograph copd-6 ${ }^{\mathrm{TM}}$ ) Hausärzte bei der Identifikation von Patienten mit Verdacht auf COPD unterstützen kann.

Methodik: Bei 17856 Patienten > 40 Jahre (Raucher/Exraucher mit Husten u./o. Belastungsatemnot) wurden durch Hausärzte mit dem Lungenfunktions-Schnellmessgerät präbronchodilatatorisch $\mathrm{FEV}_{1}\left[\%\right.$ v. Soll] und $\mathrm{FEV}_{1} / \mathrm{FEV}_{6}$ gemessen. Darüber hinaus füllte der Hausarzt einen Fragebogen zu Symptomen, Anamnese und geplanten Maßnahmen aus und gab seine Einschätzung hinsichtlich eines eventuell bestehenden COPD-Verdachts an.

Ergebnisse: 2927 Patienten (16,7\%) wiesen ein $\mathrm{FEV}_{1} / \mathrm{FEV}_{6}<70 \%$ auf; $88,2 \%$ davon wurden vom Arzt als COPD-Verdachtspatienten eingestuft. Die Gesamtzahl aller Patienten mit COPD-Verdacht gemäß der klinischen Einschätzung der Ärzte war deutlich größer (10000; 56\% der Gesamtpopulation); nur bei 25,3\% von ihnen war ein $\mathrm{FEV}_{1} /$ $\mathrm{FEV}_{6}<70 \%$ dokumentiert. Patienten, die trotz $\mathrm{FEV}_{1} / \mathrm{FEV}_{6} \geq 70 \%$ als COPD-Verdachtspatienten bewertet wurden, waren - verglichen mit Patienten ohne COPD-Verdacht - häufiger männlich, hatten mehr Zigaretten-Packungsjahre, als Hauptsymptom häufiger Dyspnoe, jedoch seltener Husten. Sie hatten mehr Begleiterkrankungen und vorausgegangene Krankenhausaufenthalte, mehr Verordnungen von Bronchodilatatoren, Glukokortikoiden und Antibiotika im vergangenen Jahr sowie niedrigere $\mathrm{FEV}_{1}$-Werte. Bei $61,3 \%$ der COPD-Verdachtspatienten plante der Hausarzt eine Abklärung mittels Spirometrie, bei 39,9\% alternativ oder zusätzlich die Vorstellung bei einem Pneumologen.

\section{Abstract \\ $\nabla$}

Background: Screening measures can facilitate the diagnosis of chronic obstructive pulmonary disease (COPD) and help save costs and time. We examined whether use of a lung function screener (Vitalograph copd-6 ${ }^{\mathrm{TM}}$ ) can help general practitioners to identify patients at risk for COPD.

Methods: In 17,856 patients aged $>40$ years (smokers/ex-smokers with cough and/or exertional dyspnoea) general practitioners measured prebronchodilator $\mathrm{FEV}_{1}$ [\% of predicted] and $\mathrm{FEV}_{1} / \mathrm{FEV}_{6}$ with the lung function screening device. In addition, the general practitioners completed a questionnaire on symptoms, history and planned measures and estimated whether or not the patient was at risk for COPD.

Results: In 2927 patients (16.7\%) an $\mathrm{FEV}_{1} / \mathrm{FEV}_{6}$ $<70 \%$ was measured; $88.2 \%$ of these were classed by the doctors as being at risk for COPD. The total number of all patients with suspected COPD was considerably greater $(10,000 ; 56 \%$ of the total population); in only $25.3 \%$ was an $\mathrm{FEV}_{1} / \mathrm{FEV}_{6}<70 \%$ documented. Compared with patients without a suspicion of COPD, patients judged to be at risk for COPD in spite of an $\mathrm{FEV}_{1} / \mathrm{FEV}_{6} \geq 70 \%$ were more often male, had more cigarette pack years and more often had dyspnoea, but less often cough, as main symptom. They had more concomitant diseases and previous hospitalisations, more prescriptions for bronchodilators, glucocorticoids and antibiotics in the past year and lower $\mathrm{FEV}_{1}$ values. In $61.3 \%$ of the patients with suspected COPD the general practitioners planned further evaluation by spirometry, in $39.9 \%$ referral to a pulmonologist as alternative or additional procedures were suggested.

Conclusion: Most patients with an $\mathrm{FEV}_{1} / \mathrm{FEV}_{6}$ $<70 \%$ measured with the lung function screener Vitalograph copd- $6^{\mathrm{TM}}$ were classed by the general practitioners as being at risk for COPD. 
Schlussfolgerung: Hausärzte nahmen bei einem mittels des Lungenfunktions-Schnellmessgeräts „Vitalograph copd-6 TM“ gemessenen $\mathrm{FEV}_{1} / \mathrm{FEV}_{6}<70 \%$ überwiegend einen COPD-Verdacht an. Auch bei unauffälligen $\mathrm{FEV}_{1} / \mathrm{FEV}_{6}$-Werten wurde häufig eine COPD-Verdachtsdiagnose gestellt, wenn klinische Merkmale des Patienten oder ein reduziertes $\mathrm{FEV}_{1}$ auf einen solchen Verdacht hinwiesen.

\section{Einleitung \\ $\nabla$}

Die COPD ist weltweit eine der häufigsten Ursachen für chronische Immobilität und Mortalität. Nach Schätzungen der WHO wird COPD bis zum Jahr 2030 weltweit die dritthäufigste Todesursache sein [1]. In Deutschland sind derzeit etwa 6,8 Millionen Menschen an einer COPD erkrankt [2]. Der Verlauf der COPD ist durch eine progrediente Verschlechterung der Lungenfunktion und durch eine zunehmende Verschlechterung des Allgemeinbefindens, der Leistungsfähigkeit und der Lebensqualität gekennzeichnet. Eine frühe Diagnostik sowie gegebenenfalls frühzeitig einsetzende medikamentöse Maßnahmen sind von entscheidender Bedeutung beim Management der COPD und tragen zur Verringerung von Komplikationen wie z. B. rezidivierende Exazerbationen bei $[3,4]$. In der Praxis hingegen ist die COPD noch häufig unterdiagnostiziert. Eine Stichprobenuntersuchung bei Teilnehmern über 40 Jahren, die im Jahre 2005 im Raum Hannover durchgeführt wurde, ergab, dass 13,2\% aller Teilnehmer die Lungenfunktions-Kriterien für eine COPD-Diagnose erfüllten, während eine solche Diagnose hingegen nur bei 7,7\% der Teilnehmer vorlag [5].

Zur Diagnose der COPD durch eine Lungenfunktionsuntersuchung sowie zur Bestimmung des Krankheitsschweregrades ist die Spirometrie heute die am besten validierte Methode und gemäß den aktuellen Leitlinien eine notwendige Voraussetzung für die Diagnose einer COPD. Die Spirometrie erfordert eine intensive Schulung, die jedoch in der Praxis häufig nicht ausreichend umgesetzt werden kann [6].

Aus diesem Grund wurden in den letzten Jahren eine Reihe von Lungenfunktions-Schnellmessgeräten entwickelt, die einfach und rasch anwendbar sind und eine Früherkennung von COPDPatienten unterstützen sollen. Eines dieser Geräte ist das digitale Lungenfunktions-Schnellmessgerät „Vitalograph copd-6 ${ }^{\mathrm{TM}}$ “, das die Messung der Lungenfunktionsparameter FEV $_{1}$ und FEV $_{6}$ ermöglicht. Der $\mathrm{FEV}_{6}$-Wert wird dabei als Surrogatparameter für die Vitalkapazität verwendet.

Studien mit dem „Vitalograph copd-6 ${ }^{\mathrm{TM}}$ “ oder einem ähnlichen Lungenfunktions-Schnellmessgerät haben die Zuverlässigkeit dieser Geräte im Vergleich zur Spirometrie untersucht. Bei kontrollierten Studien mit bis zu etwa 1000 Patienten ergaben sich dabei für die Lungenfunktions-Schnellmessgeräte Sensitivitätswerte von 79-85\% und Spezifitätswerte von 80-95\% im Bezug auf die erfolgreiche Detektion einer COPD-Erkrankung im Vergleich zum Goldstandard Spirometrie [7-9].

Erkenntnisse zur Durchführbarkeit von COPD-Screeninguntersuchungen mit Lungenfunktions-Schnellmessgeräten unter Praxisbedingungen bei größeren Patientenkollektiven in Deutschland sowie zur Akzeptanz dieser Untersuchungen durch Hausärzte fehlen bisher. Wir untersuchten daher, in welchem Ausmaß die Anwendung des „Vitalograph copd-6 ${ }^{\text {TM“", }}$ kombiniert mit einem standardisierten Fragebogen zur Anamnese und Symptomatik, die Einschätzung von Hausärzten zum Vorliegen einer COPD-Verdachtsdiagnose unterstützen kann.
Even in patients with unremarkable $\mathrm{FEV}_{1} / \mathrm{FEV}_{6}$ values the diagnosis of suspected COPD was often made if clinical signs or symptoms or a reduced $\mathrm{FEV}_{1}$ pointed to such a suspicion.

\section{Methodik \\ $\nabla$}

Hausarztpraxen (Internisten, Allgemeinärzte, Praktische Ärzte) rekrutierten Patienten beiderlei Geschlechts (maximal 5 Patienten pro Arztpraxis). Als Einschlusskriterien waren dabei ein Alter $\geq 40$ Jahre, ein positiver Raucherstatus (aktuelle Raucher oder Exraucher) und die Angabe von regelmäßigem oder wiederholtem Husten und/oder Atemnot festgelegt worden.

Mithilfe des Lungenfunktions-Schnellmessgeräts „Vitalograph copd-6 ${ }^{\text {TM" }}$ (Vitalograph Ltd., Maids Moreton Buckingham, England; www.vitalograph.de) wurden $\mathrm{FEV}_{1}[\%$ v. Soll] und das $\mathrm{FEV}_{1} / \mathrm{FEV}_{6}$-Verhältnis ermittelt. Gemäß den Angaben des Herstellers wurde der Patient dafür aufgefordert, für 6 Sekunden so stark und schnell wie möglich in das Mundstück des Geräts auszuatmen. Dieses Manöver wurde jeweils dreimal wiederholt und das beste Ergebnis vom Gerät angezeigt. Alle Messungen wurden präbronchodilatatorisch durchgeführt, also ohne vorherige Anwendung eines Bronchodilatators. Das LungenfunktionsSchnellmessgerät bewertete ein $\mathrm{FEV}_{1} / \mathrm{FEV}_{6}$-Verhältnis $<70 \%$ als auffällig. Ein $\mathrm{FEV}_{1} / \mathrm{FEV}_{6}$-Verhältnis $\geq 70 \%$ wurde auf dem Anzeigefeld des Geräts als normal bewertet. Das $\mathrm{FEV}_{1}$ wird vom Gerät als „normal“ $\left(\mathrm{FEV}_{1} \geq 80 \%\right)$ oder als reduziert $\left(\mathrm{FEV}_{1}<80 \%\right)$ klassifiziert. In den Verwendungshinweisen vermerkt der Hersteller dazu, dass eine COPD bei normalem $\mathrm{FEV}_{1} / \mathrm{FEV}_{6}$ ausgeschlossen werden könne; bei reduziertem $\mathrm{FEV}_{1}$ und gleichzeitig normalem $\mathrm{FEV}_{1} / \mathrm{FEV}_{6}$ sei eine COPD unwahrscheinlich, eine Spirometrie wird aber in diesem Fall zur weiteren Abklärung empfohlen.

Für jeden Patienten dokumentierte der Hausarzt neben den mittels des „Vitalograph copd-6 ${ }^{\text {TM“ }}$ erhobenen Lungenfunktionswerten mithilfe eines standardisierten Fragebogens auch demografische und anamnestische Daten und gab an, ob aus seiner Sicht ein COPD-Verdacht vorlag oder nicht. Abgefragt wurden im Einzelnen Angaben zu aktuellen Beschwerden, zum Raucherstatus, zu Begleiterkrankungen, zu vorhergegangenen Verschreibungen oder Krankenhauseinweisungen aufgrund von respiratorischen Beschwerden und zu geplanten weiteren Maßnahmen.

Die Darstellung der Ergebnisse erfolgt deskriptiv. Angegeben werden Mittelwerte \pm Standardabweichung.

\section{Ergebnisse \\ $\nabla$}

17856 Patienten (56\% männlich; 50\% aktive Raucher; mittleres Alter 57,8 $\pm 12,1$ Jahre; mittlerer Body-Mass-Index 27,1 \pm $4,9 \mathrm{~kg} / \mathrm{m}^{2}$ ) wurden in insgesamt 3675 hausärztlichen Praxen eingeschlossen.

Eine auffällige $\mathrm{FEV}_{1} / \mathrm{FEV}_{6}$-Ratio $<70 \%$ wurde vom Lungenfunktions-Schnellmessgerät bei 2978 Patienten (16,7\%) angezeigt, ein normales $\mathrm{FEV}_{1} / \mathrm{FEV}_{6} \geq 70 \%$ bei 12038 Patienten (67,4\%) (fehlende Angabe: 15,9\%; $n=2840$ ). Ein COPD-Verdacht wurde vom Arzt bei 10000 Patienten (56,0\%) angegeben, kein COPD-Verdacht bei 7061 Patienten (39,5\%) (fehlende Angabe: 4,5\%, n= 795). 
Bei 9 von 10 Patienten mit auffälligem $\mathrm{FEV}_{1} / \mathrm{FEV}_{6}$ - aber auch bei fast jedem zweiten Patienten mit normalem $\mathrm{FEV}_{1} / \mathrm{FEV}_{6} \geq 70 \%$ lag nach Ansicht der Ärzte ein COPD-Verdacht vor. Unter den 10000 Patienten mit COPD-Verdacht lag doppelt so häufig ein normales $\mathrm{FEV}_{1} / \mathrm{FEV}_{6}$-Verhältnis vor ( $\mathrm{n}=5669$ vs. $\mathrm{n}=2627$; fehlende Daten: $n=1704$ ) ( $\bullet$ Abb. 1).

Etwa die Hälfte aller Patienten $(n=8807$; fehlende Daten: $n=$ 1810 ) hatten ein auffälliges $\mathrm{FEV}_{1}<80 \%$. Bei Patienten mit einem reduzierten $\mathrm{FEV}_{1}<80 \%$ wurde in $83,4 \%$ auch ein COPD-Verdacht von Seiten der Ärzte angegeben; hingegen wurde bei einem unauffälligen $\mathrm{FEV}_{1}$ nur in 23,8\% ein Verdacht auf COPD vermerkt.

Ein hoher Anteil der Patienten von knapp 50\% mit normalem $\mathrm{FEV}_{1} / \mathrm{FEV}_{6} \geq 70 \%$ hatte ein reduziertes $\mathrm{FEV}_{1}(<80 \%$ ). Dabei war das FEV ${ }_{1}$ bei Patienten, bei denen der Arzt trotz eines normalen $\mathrm{FEV}_{1} / \mathrm{FEV}_{6}$ einen COPD-Verdacht annahm, deutlich häufiger auffällig reduziert $\left(\mathrm{FEV}_{1}<80 \%\right)$ als bei Patienten ohne COPD-Verdacht (74,9\% vs 20,4\%) ( $\bullet$ Abb. 2 ).

Patienten mit COPD-Verdacht und unauffälligem $\mathrm{FEV}_{1} / \mathrm{FEV}_{6} \geq 70 \%$ litten häufiger unter dem Hauptsymptom Dyspnoe (einschließlich verminderter Belastbarkeit) als unter Husten. Bei Patienten ohne COPD-Verdacht wurden vergleichbar häufig Dyspnoe und Husten als Hauptsymptom angegeben ( $\bullet$ Tab. 1$)$. In der Gruppe der Patienten mit normalem $\mathrm{FEV}_{1} / \mathrm{FEV}_{6} \geq 70 \%$ wiesen Patienten mit COPD-Verdacht erheblich mehr Zigaretten-Packungsjahre auf als Patienten ohne COPD-Verdacht (30,73 $\pm 29,95$ vs. 21,64 \pm 17,33), obwohl der Anteil aktiver Raucher nur geringfügig höher war: $53,4 \%$ vs. $46,3 \%$. Auch fanden sich bei diesen Patienten häufiger chronische Herzerkrankungen und Diabetes mellitus, aber seltener Allergien als Begleiterkrankungen. Darüber hinaus hatten sie in den vorhergegangenen 12 Monaten häufiger Verschreibungen von Bronchodilatatoren und Kortikosteroiden erhalten und waren häufiger aufgrund von Atembeschwerden ins Krankenhaus eingewiesen worden als Patienten, bei denen bei normalen $\mathrm{FEV}_{1} / \mathrm{FEV}_{6}$ kein Verdacht auf COPD angenommen wurde ( Tab. 1$)$.

Bei Patienten mit COPD-Verdacht wurde überwiegend die diagnostische Abklärung durch Spirometrie und/oder fachärztliche

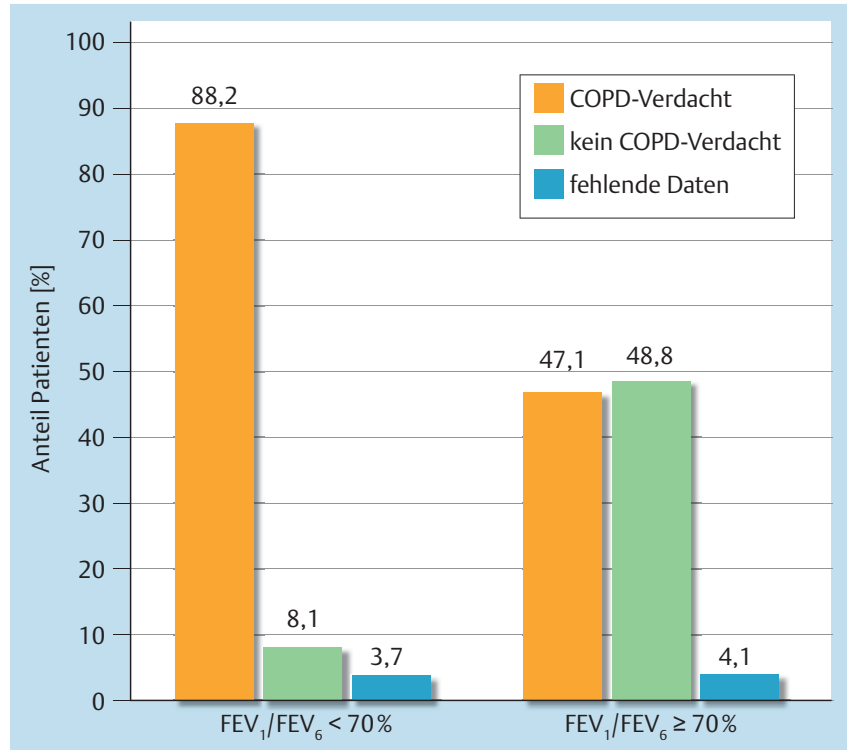

Abb. 1 COPD-Verdacht bei Patienten mit $\mathrm{FEV}_{1} / \mathrm{FEV}_{6}<$ oder $\geq 70 \%$. FEV 1 [\% v. Soll] und das $\mathrm{FEV}_{1} / \mathrm{FEV}_{6}$-Verhältnis wurden präbronchodilatatorisch mithilfe des Lungenfunktions-Schnellmessgeräts „Vitalograph copd-6 ${ }^{\mathrm{T} M “}$ ermittelt. Auf der Basis der Lungenfunktionswerte und der anamnestischen Angaben sowie der Symptomatik des Patienten gab der Hausarzt an, ob aus seiner Sicht ein COPD-Verdacht vorlag oder nicht. Angegeben ist der jeweilige Anteil der Patienten in \%.

Untersuchung geplant. Diese Entscheidung war dabei nur bedingt abhängig vom Ergebnis des Lungenfunktions-Schnellmessgeräts: Auch bei einem normalen Messergebnis $\left(\mathrm{FEV}_{1} /\right.$ $\mathrm{FEV}_{6} \geq 70 \%$ ) wurden weitere diagnostische Maßnahmen geplant, wenn der Arzt aufgrund der Anamnese und der Symptomkonstellation einen COPD-Verdacht hatte: Bei 37,4\% dieser Patienten wurde eine Überweisung zum Pneumologen und/oder in 60,4\% der Fälle eine Spirometrie geplant. Bei Patienten hingegen, bei denen aus ärztlicher Sicht kein COPD-Verdacht bestand, wurde nur selten eine weitere diagnostische Abklärung angestrebt (in

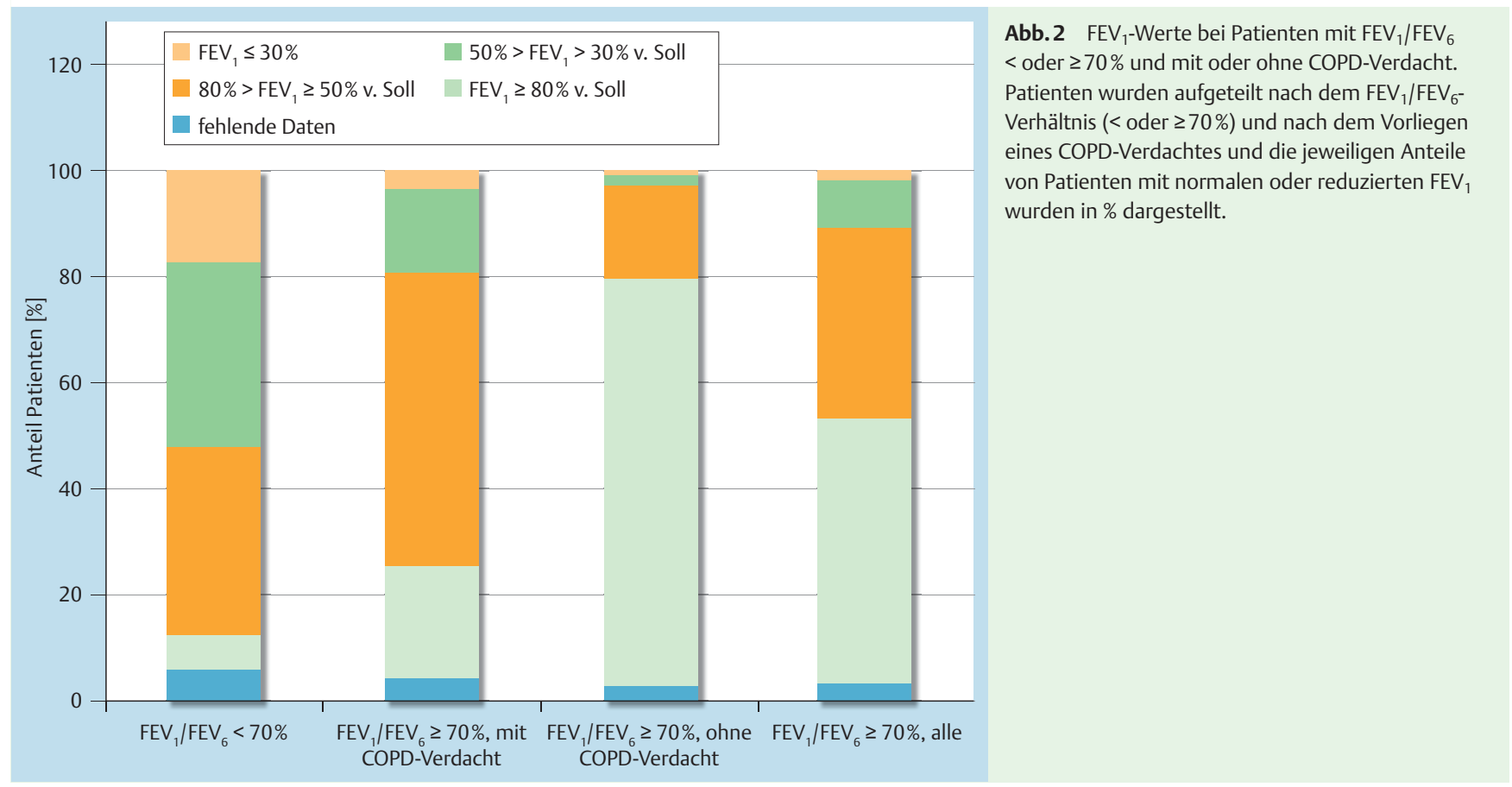


Tab. 1 Demografische Daten und anamnestische Angaben. Demografische Daten und anamnestische Angaben wurden mit Hilfe eines standardisierten Fragenbogens durch den Hausarzt dokumentiert. Das Alter und die Packungsjahre sind Mittelwerte + /- Standardabweichungen; alle anderen Angaben sind in \%.

\begin{tabular}{|c|c|c|c|c|c|}
\hline Parameter & Kategorie & $\begin{array}{l}\mathrm{FEV}_{1} / \mathrm{FEV}_{6}<70 \% \\
{[\mathrm{n}=2978]}\end{array}$ & $\begin{array}{l}\mathrm{FEV}_{1} / \mathrm{FEV}_{6} \geq 70 \% \\
\text { mit COPD-Verdacht } \\
{[\mathrm{n}=5669]}\end{array}$ & $\begin{array}{l}\mathrm{FEV}_{1} / \mathrm{FEV}_{6} \geq 70 \% \text {, } \\
\text { ohne COPD-Verdacht } \\
\text { [n=5874] }\end{array}$ & $\begin{array}{l}\mathrm{FEV}_{1} / \mathrm{FEV}_{6} \geq 70 \%, \\
{[\mathrm{n}=12038]}\end{array}$ \\
\hline \multirow[t]{2}{*}{ Geschlecht } & Männlich & 58,7 & 60,1 & 50,7 & 55,1 \\
\hline & Weiblich & 40,8 & 39,5 & 48,8 & 44,4 \\
\hline Alter & Mittelwert \pm S.A. [Jahre ] & $60,85 \pm 11,76$ & $60,19 \pm 11,76$ & $53,87 \pm 11,47$ & $56,91 \pm 12,06$ \\
\hline \multirow[t]{3}{*}{ Hauptsymptom } & $\begin{array}{l}\text { Dyspnoe (einschließlich } \\
\text { Belastungsdyspnoe) }\end{array}$ & 40,0 & 34,7 & 19,1 & 26,4 \\
\hline & Verminderte Belastbarkeit & 17,3 & 16,9 & 11,0 & 13,8 \\
\hline & Husten & 17,2 & 19,7 & 24,0 & 21,5 \\
\hline \multirow[t]{2}{*}{ Raucherstatus } & Anteil aktiver Raucher & 52,0 & 53,4 & 46,3 & 50,0 \\
\hline & Packungsjahre \pm S.A. [Jahre] & $33,06 \pm 33,86$ & $30,73 \pm 29,95$ & $21,64 \pm 17,33$ & $26,17 \pm 25,58$ \\
\hline \multirow{2}{*}{$\begin{array}{l}\text { Verordnungen } \\
\text { im letzten Jahr }{ }^{1}\end{array}$} & Bronchodilatatoren & 54,4 & 51,2 & 21,6 & 35,8 \\
\hline & Glukokortikosteroide & 44,9 & 40,1 & 16,6 & 28,0 \\
\hline \multirow[t]{3}{*}{ Begleiterkrankungen ${ }^{1}$} & chronische Herzerkrankung & 27,4 & 27,6 & 13,5 & 20,3 \\
\hline & Diabetes mellitus & 21,1 & 20,6 & 12,9 & 16,5 \\
\hline & Allergie & 19,2 & 19,8 & 28,7 & 24,4 \\
\hline $\begin{array}{l}\text { Hospitalisation } \\
\text { im letzten Jahr }\end{array}$ & $\begin{array}{l}\text { Hospitalisation aufgrund von } \\
\text { Atembeschwerden }\end{array}$ & 10,2 & 6,8 & 2,2 & 4,4 \\
\hline
\end{tabular}

${ }^{1}$ Mehrfachangaben waren möglich.

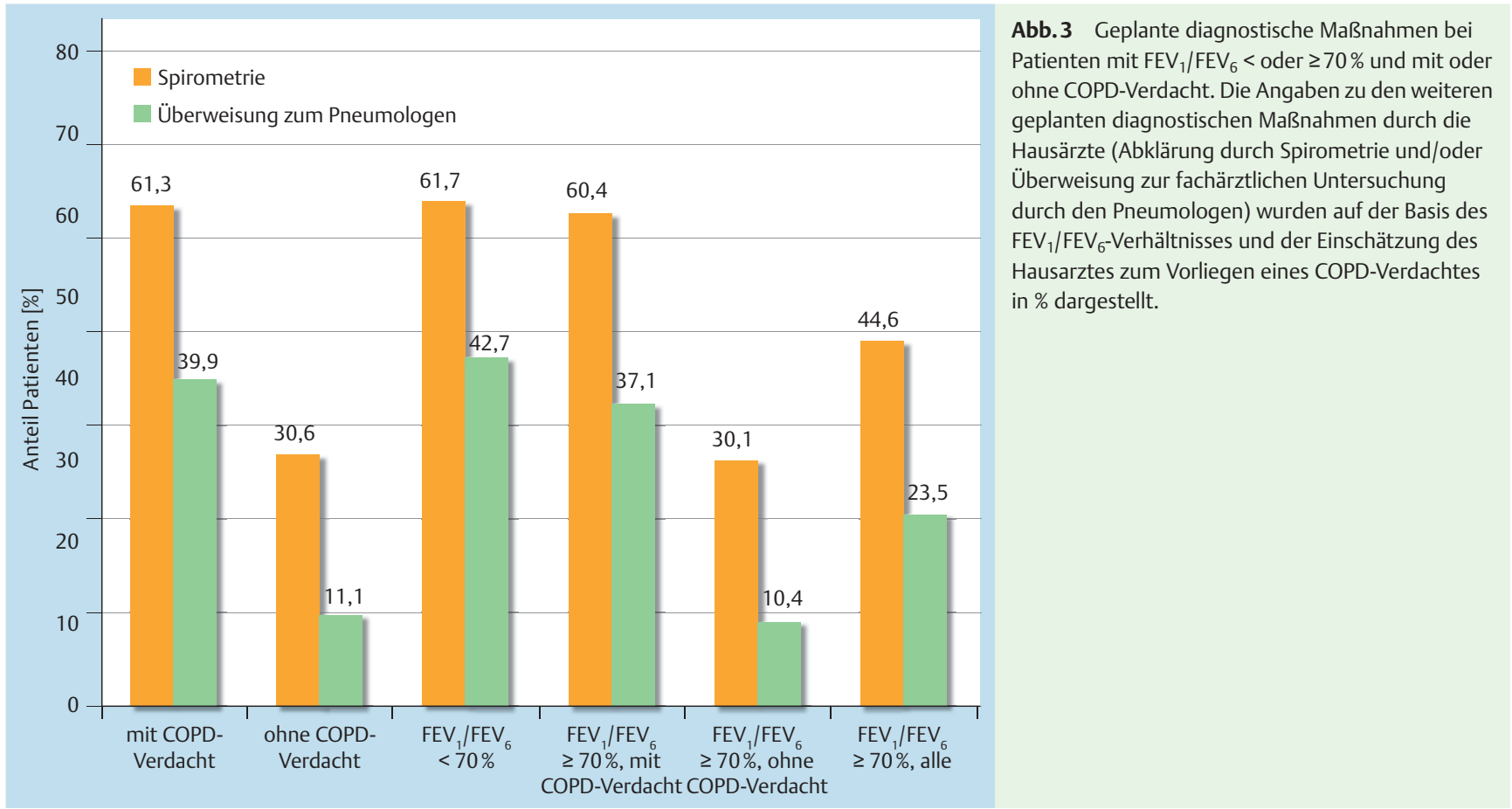

$11,1 \%$ eine Überweisung zum Pneumologen und/oder in 30,6\% eine Abklärung durch Spirometrie) ( $\bullet$ Abb.3).

\section{Diskussion}

Die Studie zeigt, dass eine Untersuchung mittels eines Lungenfunktions-Schnellmessgeräts in hausärzlichen Praxen auch bei größeren Patientenkollektiven mit COPD-Risiko durchgeführt werden kann.

Der ärztliche Verdacht auf das Vorliegen einer COPD korrelierte nur in eingeschränktem Umfang mit der Bewertung durch das
Lungenfunktions-Schnellmessgerät. So wurde ärztlicherseits dreimal häufiger ein COPD-Verdacht gestellt, als aufgrund der Bewertung des $\mathrm{FEV}_{1} / \mathrm{FEV}_{6}$-Verhältnisses durch das Schnellmessgerät abzuleiten gewesen wäre. Bei vielen Patienten fiel dabei eine Diskrepanz zwischen unauffälligem $\mathrm{FEV}_{1} / \mathrm{FEV}_{6}$-Verhältnis und reduziertem $\mathrm{FEV}_{1}$ auf. Dadurch korrelierte $\mathrm{FEV}_{1}$ für sich genommen deutlich besser mit der klinischen Einschätzung durch die Ärzte als das Verhältnis $\mathrm{FEV}_{1} / \mathrm{FEV}_{6}$. Bei mehr als 8 von $10 \mathrm{~Pa}-$ tienten mit einem erniedrigten $\mathrm{FEV}_{1}<80 \%$ gaben die Ärzten auch einen Verdacht auf COPD an, während nur bei etwa 20\% der Patienten mit einem normalen $\mathrm{FEV}_{1}$ trotzdem ein COPD-Verdacht bestand. 
Dieses Ergebnis zeigt, dass die Ärzte den Empfehlungen des Herstellers des Lungenfunktions-Schnellmessgeräts zur Beurteilung des $F E V_{1}$ und des $F E V_{1} / \mathrm{FEV}_{6}$-Quotienten nur eingeschränkt folgten und weist bei der untersuchten Population von Patienten mit Atemwegssymptomen auf eine insgesamt niedrige Akzeptanz des durch das Schnellmessgerät ermittelten $\mathrm{FEV}_{1} / \mathrm{FEV}_{6}$-Quotienten als Surrogat für $\mathrm{FEV}_{1} / \mathrm{FVC}$ bei den Ärzten hin.

Die Validität des $\mathrm{FEV}_{6}$-Wertes als Surrogat für die Vitalkapazität wird in der Literatur kontrovers diskutiert. Während die Leitlinien zurzeit weiterhin die Vitalkapazität als Standard für die Bestimmung des Obstruktionsgrades empfehlen [3,4], kommt Pedersen in einer Übersichtsarbeit zum Ergebnis, dass der $\mathrm{FEV}_{6}$ eine valide Alternative zur Vitalkapazität bei der Bestimmung des Obstruktionsgrades darstellen kann [10]. Gleichzeitig weist er aber auch auf die zentrale Bedeutung eines korrekten Referenzschwellenwerts bei der Beurteilung des $\mathrm{FEV}_{1} / \mathrm{FEV}_{6}$-Quotienten hin. Vandevoorde et al. zeigten diesbezüglich in ihrer spirometrischen Untersuchung, dass bei Anwendung des $\mathrm{FEV}_{1} / \mathrm{FEV}_{6}{ }^{-}$ Quotienten ein erhöhter Schwellenwert von 73\% eine verlässliche Alternative zur Anwendung des Schwellenwertes von 70\% beim $\mathrm{FEV}_{1} / \mathrm{FVC}$-Quotienten darstellt [11]. Mehrere kürzlich veröffentlichte Studien haben versucht, einen optimalen Schwellenwert zur Beurteilung des $\mathrm{FEV}_{1} / \mathrm{FEV}_{6}$ bei der Anwendung von Lungenfunktions-Schnellmessgeräten zu ermitteln [7,8,9]. Die Ergebnisse erlauben bisher keine finale Bewertung, aber die zwei Untersuchungen von Frith und Thorn [7,9] weisen darauf hin, dass eine Erhöhung des Schwellenwerts für den $\mathrm{FEV}_{1} / \mathrm{FEV}_{6}$ von $<70 \%$ auf $<73 \%$ bzw. $<75 \%$ die Zahl falsch-negativer Bewertungen reduzieren und damit die Sensitivität auch bei der Anwendung von Lungenfunktions-Schnellmessgeräten erhöhen könnte. Insgesamt weisen unsere Ergebnisse darauf hin, dass die Ärzte sich bei Einschätzung des COPD-Risikopotenzials ihrer Patienten eher auf die Anamnese- und Befundkonstellation beziehen als auf die durch das COPD-Schnellmessgerät ermittelten Lungenfunktionswerte. Neben klassischen Risikofaktoren wie einer ausgeprägten Raucheranamnese führten vor allem auch typische Symptome wie Dyspnoe und verminderte Belastbarkeit vermehrt zur Verdachtsdiagnose COPD. Die Einstufung von Patientensymptomen als einer zentralen Komponente bei der Beurteilung der COPD-Erkrankung durch die Ärzte liegt dabei im Einklang mit den kürzlich aktualisierten GOLD-Empfehlungen und anderen Studien, die den Stellenwert von Patientensymptomen bei der Einstufung von COPD-Schweregraden als wichtige zusätzliche Bewertungskriterien neben Lungenfunktionswerten betonen $[3,12]$.

Die hier vorgestellte Untersuchung beinhaltet mehrere Limitationen, wobei in erster Linie die fehlende spirometrische Untersuchung zur Validierung der Ergebnisse des LungenfunktionsSchnellmessgeräts genannt werden muss. Dadurch bleiben die genauen Gründe für die Diskrepanz zwischen der Einstufung durch die Ärzte und der Bewertung durch das Schnellmessgerät ungeklärt. Wie zuvor diskutiert, könnte eine mögliche Ursache darin liegen, dass die Ärzte sich in erster Linie auf ihre klinische Einschätzung verlassen und die Bewertung durch das Schnellmessgerät nur akzeptiert haben, wenn diese mit der eigenen klinischen Bewertung übereinstimmte. Darüber hinaus könnten auch technische Probleme bei der Messung zur geringen Übereinstimmung zwischen den klinischen Einschätzungen der Ärzte und der Risikobeurteilung durch das COPD-Schnellmessgerät beigetragen haben.
Darüber hinaus stellte die untersuchte Patientenpopulation ein Kollektiv mit einem erhöhten COPD-Risiko dar. In einer früheren Screening-Untersuchung haben wir gezeigt, dass die Selektion von Patienten über 40 Jahre mit positiver Raucheranamnese und aktuellem Husten bzw. Atemnotbeschwerden die Wahrscheinlichkeit einer bestätigten COPD-Diagnose auf etwa 50\% erhöht [12]. Inwieweit sich die hier vorgestellten Ergebnisse auch auf eine unselektierte Population von asymptomatischen Patienten ausweiten lassen, muss daher offen bleiben.

Die vorliegende Untersuchung hat insgesamt keine klaren Hinweise ergeben, dass die Anwendung eines LungenfunktionsSchnellmessgeräts zum COPD-Screening zur Erkennung von mehr Risikopatienten führt, als bei alleiniger ärztlicher Beurteilung der vorhandenen Befund- und Symptomkonstellation gefunden würden. Ebenso wenig konnten wir in unserer Studie Hinweise finden, dass die Anwendung eines LungenfunktionsSchnellmessgeräts in der hausärztlichen Praxis weitere diagnostische Maßnahmen vermeidet.

Es sind daher weitere Studien notwendig, um die Kriterien der COPD-Verdachtsbeurteilung durch Lungenfunktions-Schnellmessgeräte genauer zu definieren und zu validieren. Bis dahin sollte der Schwerpunkt darin liegen, die routinemäßige Anwendung und die Qualität der spirometrischen Lungenfunktionsmessung in der hausärztlichen Praxis weiter zu verbessern.

\section{Interessenkonflikte \\ $\nabla$}

Die Autoren geben an, dass kein Interessenkonflikt besteht.

\section{Literatur}

1 World Health Organization. Global Burden of Disease (GBD) 2005 Study. www.who.int

2 Pritzkuleit R, Beske F, Katalinic A. Erkrankungszahlen in der Pneumologie - eine Projektion bis 2060. Pneumologie 2010; 64: 535-540

3 Global Initiative for Chronic Obstructive Lung Disease. Global strategy for the diagnosis, management, and prevention of chronic obstructive pulmonary disease. 2011: www.goldcopd.org

4 Vogelmeier C, Buhl R, Criée CP et al. Leitlinie der Deutschen Atemwegsliga und der Deutschen Gesellschaft für Pneumologie und Beatmungsmedizin zur Diagnostik und Therapie von Patienten mit chronisch obstruktiver Bronchitis und Lungenemphysem (COPD). Pneumologie 2007; 61: e1 - e40

5 Geldmacher H, Biller $H$, Herbst $H$ et al. Die Prävalenz der chronisch obstruktiven Lungenerkrankung (COPD) in Deutschland. Ergebnisse der BOLD-Studie. Dtsch Med Wochenschr 2008; 133: 2609-2614

6 Enright $P$. The use and abuse of office spirometry. Primary Care Respiratory Journal 2008; 17: $238-242$

7 Frith P, Crockett A, Beilby J et al. Simplified COPD screening: validation of the PiKo- $6^{\circledR}$ in primary care. Prim Care Respir J 2011; 20: 190-198

8 Sichletidis L, Spyratos D, Papaioannou $M$ et al. A combination of the IPAG questionnaire and PiKo- $6^{\circledR}$ flow meter is a valuable screening tool for COPD in the primary care setting. Prim Care Respir J 2011; 20 : $184-189$

9 Thorn J, Tilling B, Lisspers Ket al. Improved prediction of COPD in at-risk patients using lung function pre-screening in primary care: a real-life study and cost-effectiveness analysis. Prim Care Respir J 2012; 21: $159-166$

10 Pedersen OF. FEV6: a shortcut in spirometry? Eur Respir J 2006; 27 : $245-247$

11 Vandevoorde J, Verbanck S, Schuermans $D$ et al. Obstructive and restrictive spirometric patterns: fixed cut-offs for $\mathrm{FEV}_{1} / \mathrm{FEV}_{6}$ and $\mathrm{FEV}_{6}$. Eur Respir J 2006; 27: 378-383

12 Koegler H, Metzdorf N, Glaab T et al. Preselection of patients at risk for COPD by two simple screening questions. Respiratory Medicine 2010; 104: $1012-1019$ 